EUROPEAN ORGANIZATION FOR NUCLEAR RESEARCH

European Laboratory for Particle Physics

Large Hadron Collider Project

LHC Project Report 503

\title{
SEU Tests Performed on the Digital Communication System for LHC Cryogenic Instrumentation
}

J. Casas-Cubillos, F. Faccio, P. Gomes, M.A. Martin, M.A. Rodriguez-Ruiz

\begin{abstract}
The future LHC particle accelerator will use a large number of cryogenic sensors and actuators, most of which are located inside the machine tunnel and therefore in a radiation environment. These elements will communicate through a fieldbus. This paper reports the irradiation study carried out on WorldFIP fieldbus communication system.

A digital communication system based on WorldFIP fieldbus protocol has been implemented and Single Event Effects (SEE) and Total Ionizing Dose (TID) radiation tests have been performed on it.
\end{abstract}

EP and LHC divisions

Published in Nuclear Instruments and Methods in Physics Research

\footnotetext{
Administrative Secretariat

LHC Division

CERN

CH - 1211 Geneva 23

Switzerland

Geneva, 17 October 2002
} 


\section{INTRODUCTION}

The future LHC machine will need more than 10000 cryogenic sensors and actuators to control the temperature of the superconductor magnets, beam screen, HTS current leads,...etc. The instrumentation is distributed more or less uniformly along the $27 \mathrm{~km}$ machine. The programmable controllers (PLCs), are clustered in protected areas (such as alcoves and IPs). The electronic system for signal conditioning and transmission will be located in crates placed under the main dipoles and will thus be exposed to the accelerator environmental radiation.

In principle, the cryogenic control system can use any of CERN's recommended fieldbuses to link sensors, actuators and PLCs, but following reports concerning the operation of WorldFIP under radiation we have decided to investigate the usability of this solution. SEE test need systematically to be performed on digital devices and systems intended to be used in radiation environments. To our knowledge both SEE and TID have never been investigated on WorldFIP with sufficient detail.

Thanks to the latest simulations of the LHC radiation environment and when the device error cross section data is available, it is possible to estimate the problems caused by SEE on the electronics placed inside the tunnel. Under the main dipoles located in the LHC regular arcs, relatively low radiation doses 2-6 Gy(Si) per year are expected and, with the exception of some critical components (mainly 14-bit ADC), it should be relatively straightforward to find radiation-tolerant components. Along the Dispersion Suppresor the doses may reach 60-180 Gy(Si) per year for which electronic components can still be used after careful selection. However around the LHC

points 1, 3, 5 and 7 the expected dose will exceed $1000 \mathrm{~Gy}(\mathrm{Si})$ per year, the degradation in the electronic components will be severe, many Components Off The Shelf (COTS) will not survive and much work is required before selecting completely all the COTS that will be installed inside the tunnel. In these difficult zones the positioning of electronic devices is critical and whenever available access galleries should be used to protect electronic equipment.

From a standardization point of view it would be desirable that all components performing a given function be identical, independently of the expected radiation doses. Whether all equipment will be designed according to the worst conditions will depend on cost optimization studies that take into account cost associated with design, tests, procurement, maintenance, etc. 


\section{WORLDFIP PROTOCOL AND MICROFIP ASIC}

WorldFIP is a fieldbus linking sensors, actuators and PLCs in industrial automation systems. MICROFIP is an ASIC implementing the WorldFIP protocol.

MICROFIP can be used either in a stand-alone mode or in tandem with a microcontroller. This paper concerns the operation in microcontrolled mode. The communication between the two elements is done through a 512-byte dual port memory. Variables and messages are stored inside this internal memory, and are accessible through a set of control and status registers. Any access is characterized by a fixed duration, which is independent from the network activity. MICROFIP can be configured with 0 to 4 "produced" variables, and 0 to 4 "consumed" variables, using a global resource capability of 15 blocks of 8 bytes each named hereafter dynamic memory. MICROFIP also has 24 bytes dedicated to configuration parameters. MICROFIP is fitted with a full transmit and receive messaging channel allowing the exchange of messages 128 bytes maximum size. MICROFIP also provides an interrupt mechanism attached to one produced variable, to the four consumed variables, and to the message transmission and reception channels.

SEE effects are expected to occur as Single Event Upsets (SEU) that corrupts the memorized data. Depending on where a SEU occurs, the MICROFIP ASIC might require the reloading of all configuration parameters before proper operation can continue (this behavior has been observed experimentally). In the experimental set-up all bytes of the memory zone for dynamic variables was investigated. It is important to indicate that no message memory has been tested because we are not intending to use this feature.

\section{RADIATION TESTS}

Only a few radiation tests per year can be performed because they are expensive and the access to the facilities is not always possible. For building a complex system, like the LHC cryogenic control system, a component pre-selection is mandatory in order to save human resources and money. This is made through the study of technical literature and device data sheets. With COTS two main 
considerations should be kept well in mind: the long-term availability of the components (the LHC will run a minimum of 10 years) and the variability of radiation performance between and within production batches.

Until now MICROFIP has been tested at TCC2 (a CERN test facility which is assumed to represent in terms of total dose and spectrum energies, the radiation environment of the LHC regular arcs in a much shorter period of time). TCC2 is an excellent facility for investigating low dose rate effects. In this facility it is difficult to perform tests dealing with SEE, for which only high-energy particles must be used.

The SEE tests have been performed at the cyclotron facility of the UCL (Universite Catholique de Louvain) in Belgium, where the proton energy and beam flux can be adjusted. The UCL cyclotron is ideal for SEU and latch-up tests on electronic devices. Heavy ion and neutron sources are also available.

Figure 1 shows the experimental set-up. A PC generates one random (or zero) byte that is sent through the FPGA to the MICROFIP 120 byte dynamic memory. The MICROFIP memory is rewritten every 10 seconds without intermediate refreshes and the memory status is checked by the PC during this time. When an error is detected, the expected and observed readouts are written into a file, including the exact memory location where the error is produced. The memory is refreshed afterwards. The power consumption is also monitored during the irradiation run.

The main task of this work is to estimate the SEU device cross section, the power consumption in function of the Total Ionizing Dose (TID) and to obtain a rough idea about the latch-up sensitivity of the system. The cross section (errors $\cdot \mathrm{cm}^{2}$ ) is calculated:

$$
\sigma=\lim _{t \rightarrow t 0} \frac{\operatorname{Er}(t)}{\Phi(t, E)}
$$

where $\operatorname{Er}(\mathrm{t})$ is the SEU error rate measured by the instrumentation and $\phi(\mathrm{t}, \mathrm{E})$ is the incident beam 
flux with energy E.

The proton beam intensity was adjusted to $2 \cdot 10^{8} \mathrm{p} \cdot \mathrm{cm}^{-2} \cdot \mathrm{s}^{-1}$ and the energy to $60 \mathrm{MeV}$. Protons induce SEU because of their nuclear interaction with the silicon nuclei in the IC substrate. The recoils from the interaction can deposit sufficient energy to induce an error. As shown in reference [1], and for an upset threshold energy typical of modern components, the SEU probability is roughly independent on the proton energy above 40-50 Mev. At these energies we can also assume that the SEU cross section is proportional to the sensitive volume of the device. Therefore the irradiation with $60 \mathrm{Mev}$ protons is representative of the expected hadron environment.

Four C131 MICROFIP boards were tested. Three of them by writing random bytes ("random test") into the MICROFIP memory and the last one with all memory bytes set to zero ("zero test"). Each board was radiated during approximately one hour. All boards survived a TID of $700 \mathrm{~Gy}(\mathrm{Si})$ and only one failed at $800 \mathrm{~Gy}(\mathrm{Si})$. One SEU on configuration registers imposing the reset of the MICROFIP unit was also observed. No latch-up was detected in any radiated unit.

SEE tests were performed in previous radiation campaigns on another two MICROFIP boards. Only two of the 15 variable's memory blocks were checked but the results obtained are in agreement with what is reported in this paper.

Cumulated ionization creates net positive trapped charges in the silicon oxide that induce leakage currents on electronic devices, such effect is shown in figure 2 by the net increase of current consumption versus the TID on the tested boards. Among the irradiated devices (figure 2) MICROFIP ASIC is the most affected by TID (Generally bipolar devices like the driver are more radiation hardened than MOS to TID). Direct consequence of leakage currents is the increment of power consumption leading to higher operating temperature thus reducing the lifetime of the component.

Figure 3 shows the number of errors detected versus the proton fluency for each board tested. As expected the detected errors depends mainly on the fluency and not on the TID. The almost linear behavior obtained is an indication of the excellent stability and homogeneity of the proton beam 
source. The probability of "0" to " 1 " SEU bit transitions is much higher than"1" to "0", the experimental data indicates a distribution of such SEU of $97 \%$ and $3 \%$ respectively. This can be seen in figure 4 where the MICROFIP with all bits set to zero ("zero test") exhibit a number of errors about twice as high as when using random words ("random test").

The SEU error cross section is calculated by using Figure 3. The highest cross section (most adverse) obtained was $\sigma=104 \cdot 10^{-12}$ errors $\cdot \mathrm{cm}^{2}$. In Figure 2 and 3 the differences between the experimental curves could be explained by the spread of characteristics between devices from the same batch or from batch to batch in COTS.

Figure 4 shows in 3D the number of errors detected through MICROFIP dynamic memory indicating the exact byte and bit position. The figures 5 and 6 shown the projection of the errors only in terms of bits and bytes position respectively. The distribution of errors through the total memory is more or less uniform.

\section{SEU PREDICTIONS FOR THE LHC}

To predict the number of errors in our communication system, we need to know the device cross section $\sigma\left(\right.$ errors.cm $\left.{ }^{2}\right)$, the LHC accelerator particle flux and energy distribution and the processing time. From the experimental results $\sigma=104 \cdot 10^{-12}$ errors $\cdot \mathrm{cm}^{2}$. At energies of few tens of Mev there is no reason to expect significant differences for proton and neutrons SEU rates [1], the main difference is the proton low energy coulomb repulsion. Which mainly decreases the inelastic cross section of the proton at low energies. For practical reasons we have considered that all hadrons with E $>20 \mathrm{Mev}$ have the same probability to produce an upset as protons with energy of $60 \mathrm{Mev}$. Then

from simulations of the regular arcs the annual fluence of hadrons with $\mathrm{E}>20 \mathrm{Mev}$ is $2.16 \cdot 10^{10} \mathrm{~h} \mathrm{~cm}^{-2}$ [2]. The total errors per year considering the 3200 transmission modules will be $E r=3200 \cdot \sigma \cdot\left(2.16 \cdot 10^{10}\right) \cong 7188$ errors per year (2 errors per year and transmission unit). By using 
memory refreshment strategies the total number of SEU errors can be reduced to 0.1 error per node per year on the regular arcs. To estimate the SEU induced errors in other accelerator points it is necessary to know with adequate precision the particle spectrum and fluency.

\section{CONCLUSIONS}

After the good results in TCC2 radiation test on C131 board, we have decided to investigate the usability of MICROFIP (WorldFIP protocol) as possible fieldbus solution. MICROFIP satisfy our needs in terms of available memory and galvanic isolation. MICROFIP working in microcontrolled mode was tested and its SEE radiation effects were studied. From the experimental data we have deduced the SEU cross section $(\sigma)$ that permit to estimate the number of SEU errors in our communication system once the LHC particles fluency and energy spectrum are known.

As for TID the tests show proper operation up to $700 \mathrm{~Gy}(\mathrm{Si})$. However it is important to note that the irradiation time is relatively short preventing self-healing of the ICs by annealing effects.

At present the most critical component required by the LHC cryogenic control system is the 14-bit ADC. Next year the first ADCs COST candidates will be radiated along-side other logical (FPGA) and analog electronic integrated circuits.

\section{ACKNOWLEDGEMENTS}

We thanks to J.L. Gomez Costa for fruitful discussion on MICROFIP C131 board. We are grateful also to R. Brun and D. Glenat for FIPDESIGNER help. 


\section{REFERENCES}

[1] M. Huhtinen, F. Faccio, Computational method to estimate Single Event Upset rates in an accelerator environment, Nuclear Instruments \& Methods in Physics Research, Elsevier 14, December 1999.

[2] G.R. Stevenson, C.A. Fynbo, The LHC machine radiation environment, CERN 10-12 April, 2000.

[3] G.C. Messenger, M.S. Ash, Single Event Phenomena, Chapman \& Hall, 1997.

[4] G.C. Messenger, M.S. Ash, The effe cts of radiation on electronic systems, VAN NOSTRAND REINHOLD, N.Y. 1992.

[5] D. Glenat, R. Brun, Résultats et remarques sur les tests en radiation d'un module WORLDFIP industriel (GESSCD-1137) adapté à l'environnement radioactif du LHC. CERN 1999. 

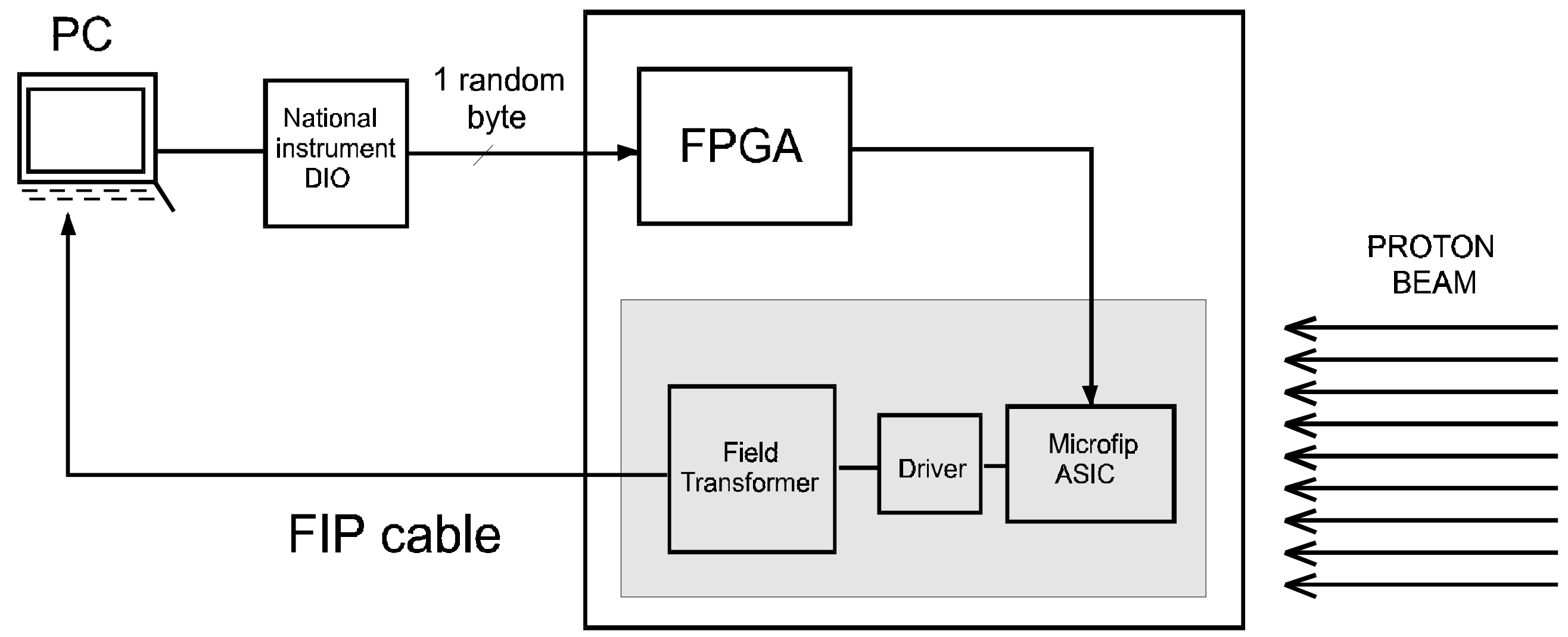


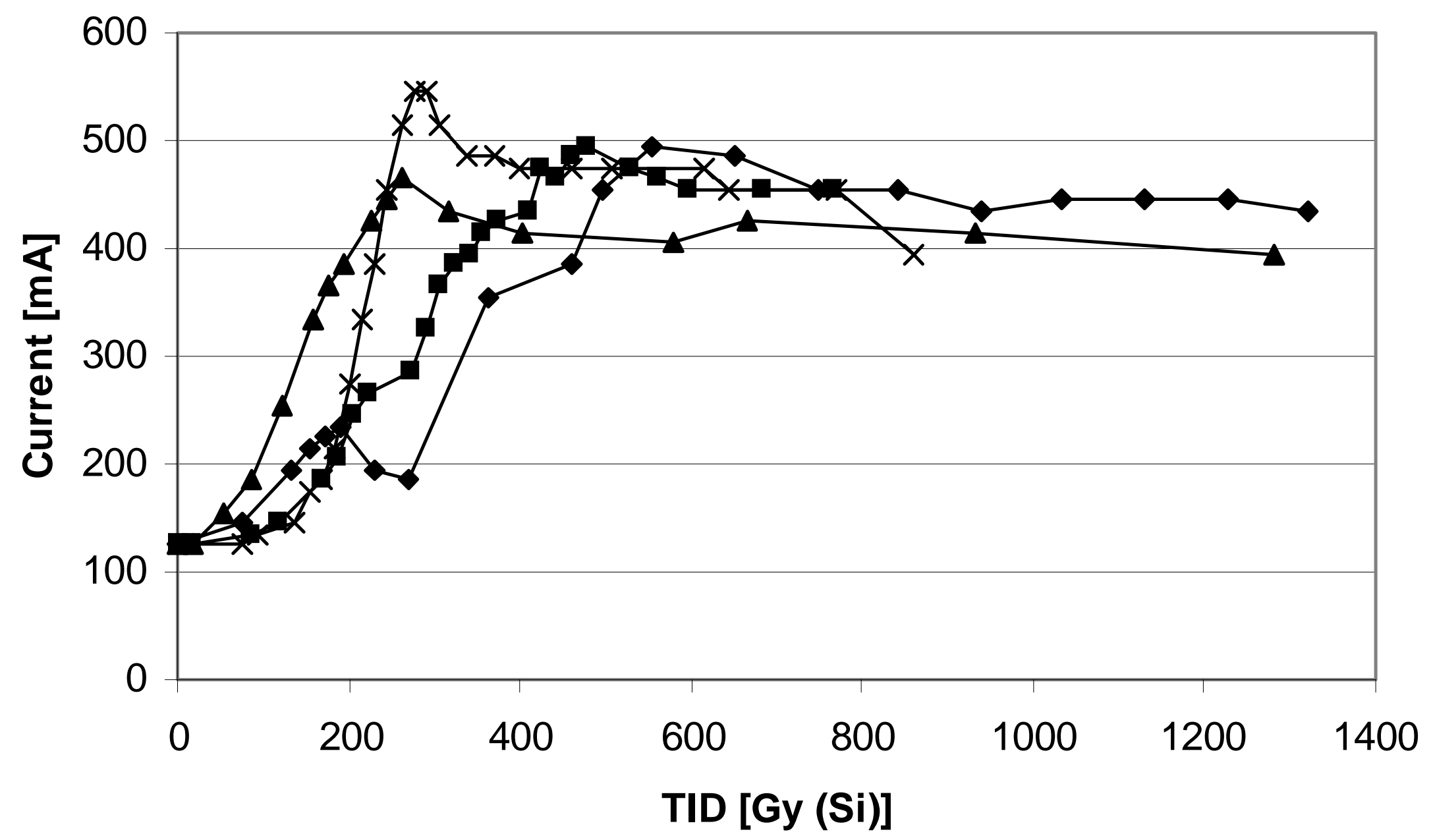

Figure 2: Boards current assumption versus the total ionizing dose 


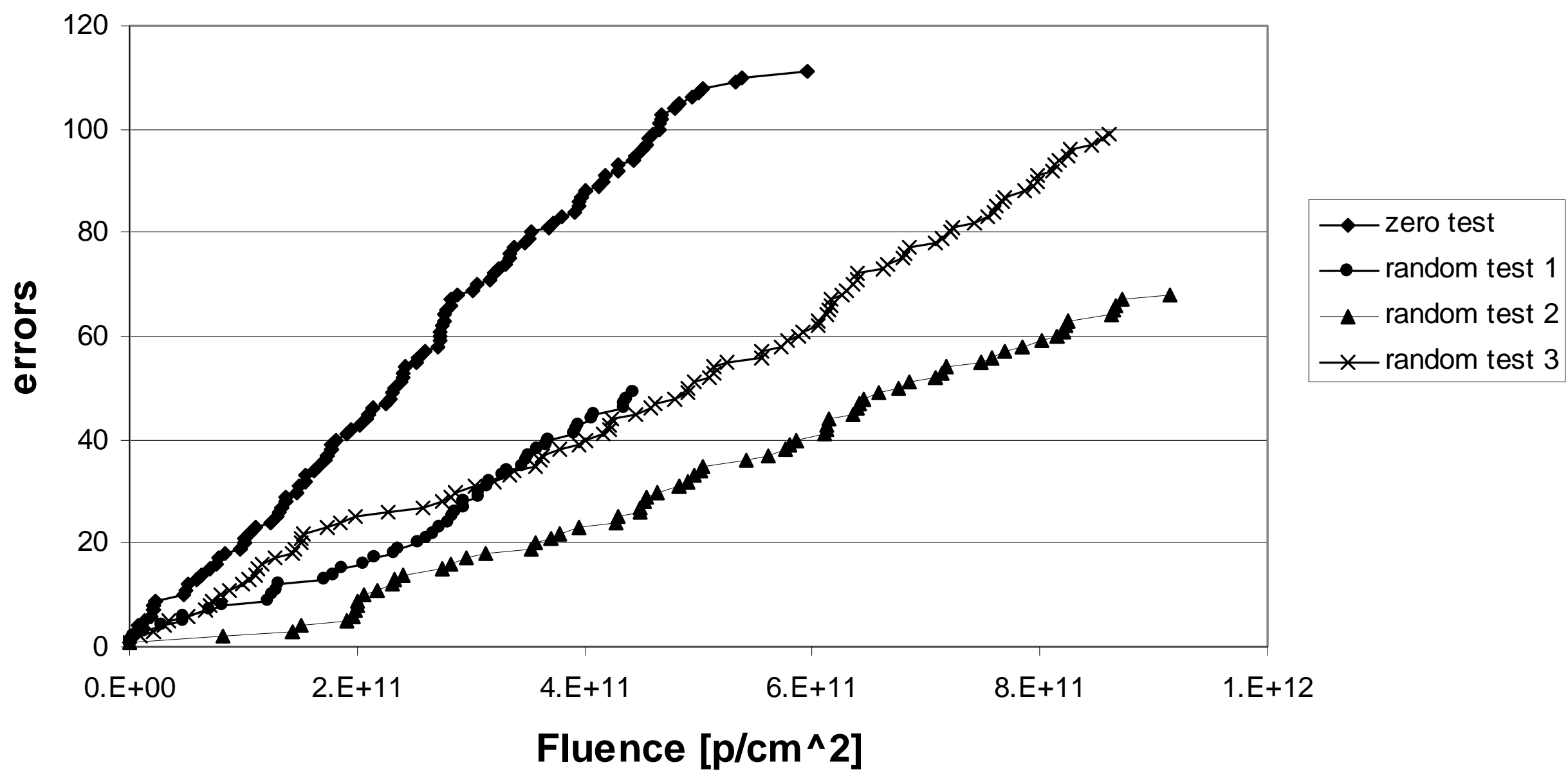

Figure 3: Number of single event upset errors detected versus the proton fluency 


\section{bit error frequency}

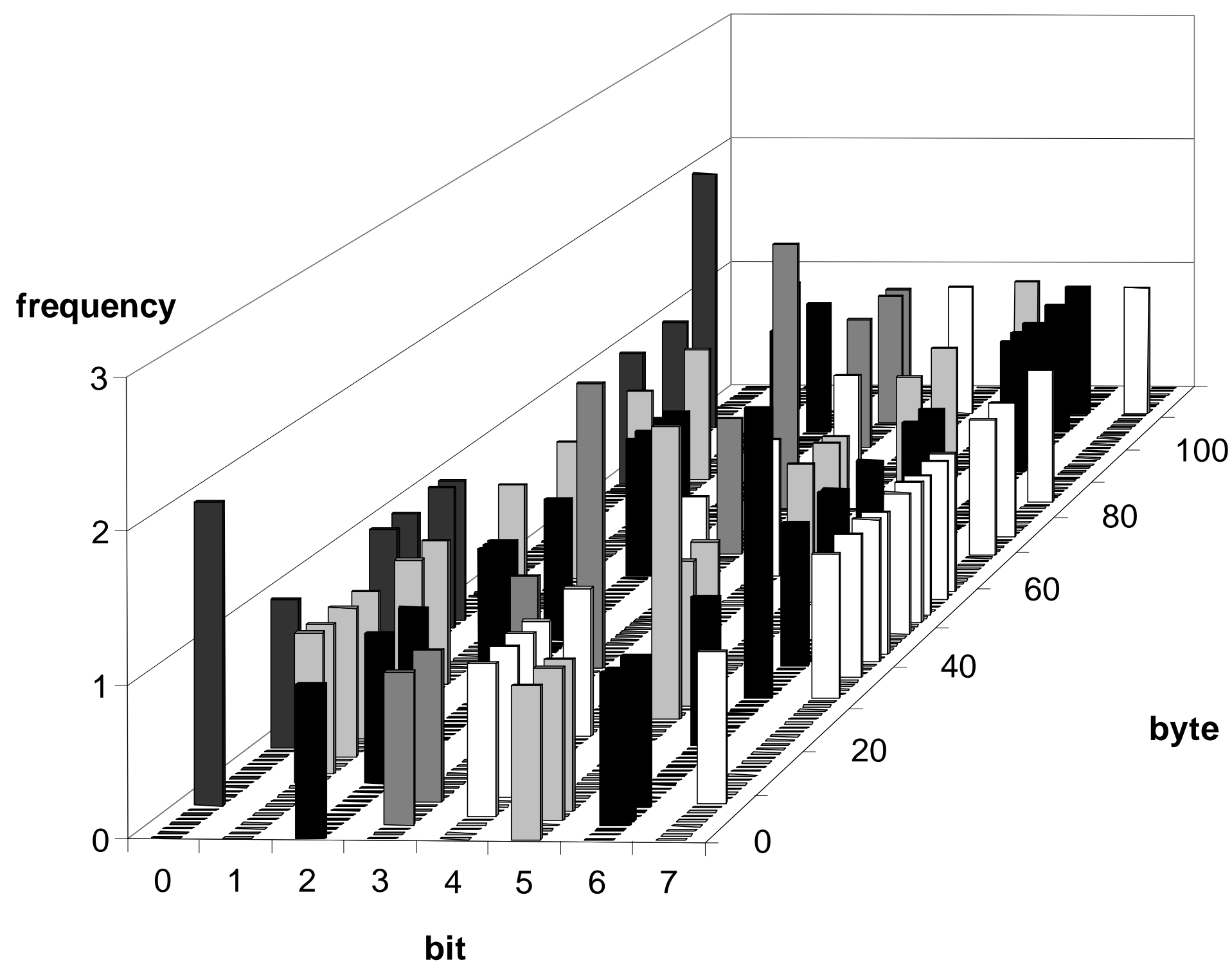




\section{bit error frequency}

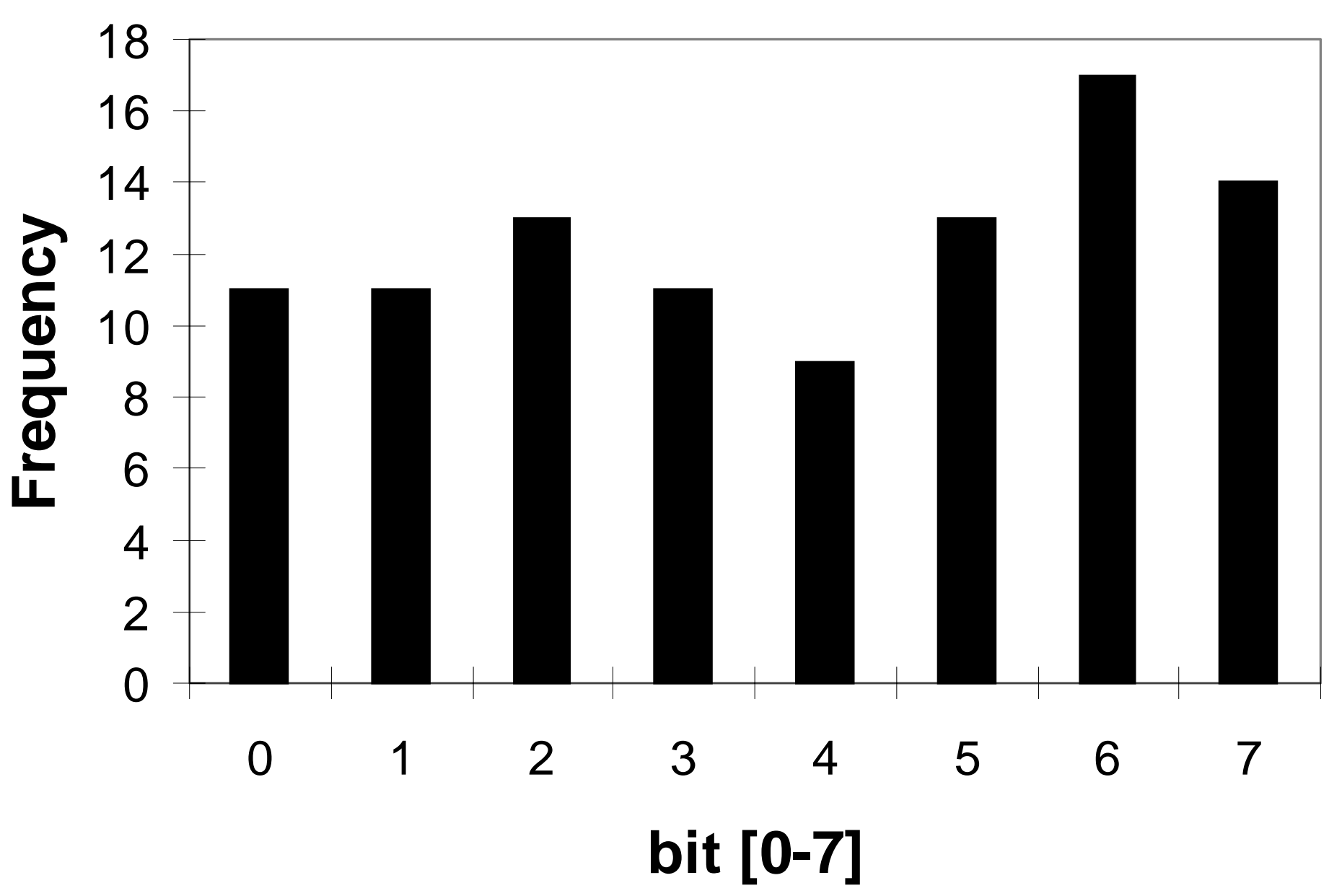

Figure 5: Projection of the single event upset errors detected in terms of bit position 


\section{byte error frequency}

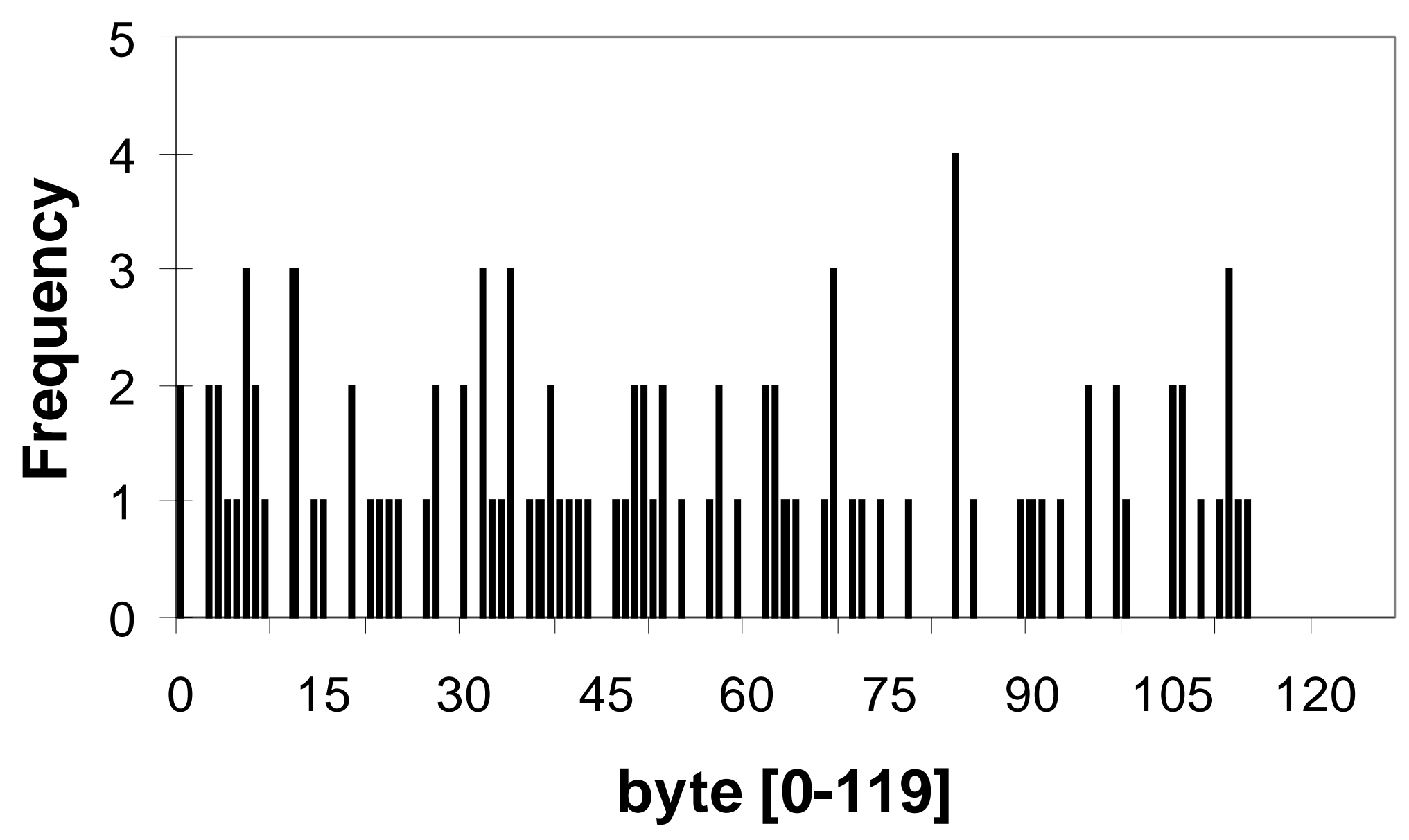

Figure 6: Projection of the single event upset errors detected in terms of byte position 